\title{
Universities and Schools Offering Forest Sector-related Programs Universités et écoles et leurs programmes relatifs au secteur forestier
}

Labour Market Development Branch/Canadian Forestry Service/ August 1988
Direction du développement du marché du travail/Service canadien des forêts/août 1988 


\section{Universities and Schools Offering Forest Sector-related Programs Universités et écoles et leurs programmes relatifs au secteur forestier}

\section{Introduction}

As a tool for students and other interested persons in following a career in forestry, the Canadian Forestry Service compiled a list of educational establishments that offer forestry or forestry-related programs. Throughout Canada, some 50 institutions offer more than 55 post-secondary educational programs dealing with the forest sector. This list includes only those programs of one year or more.

For more information, please contact the Forest Labour Market Development office of the Canadian Forestry Service at (819) 997-1107. For additional copies, please send your request to the following address: Canadian Forestry Service, Communications Branch, Ottawa K1A 1 G5

\section{Explanatory Notes}

- While recognizing the importance of the numerous programs offered in high schools, only the post-secondary programs of at least one-year duration are included for the purpose of this document. The programs are presented on a province-by-province basis, in chart form, indicating the types of programs and where they are offered. Different options within a program are not normally included.

- The numbers appearing across the top of the charts correspond to the universities and schools offering forest sector-related programs.

\section{Introduction}

Afin d'aider les étudiants(tes) et les personnes intéressées dans leur choix de carrière, le Service canadien des forêts a élaboré une liste d'institutions scolaires qui offrent des programmes en foresterie ou dans des matières reliées au domaine forestier. $\hat{A}$ travers le Canada près de 50 institutions offrent plus de 55 programmes post-secondaires dans le secteur forestier. Soulignons que seuls les programmes d'une durée d'au moins un an ont été retenus.

Pour plus de détails, vous pouvez communiquer avec le bureau du Développement du marché du travail du Service canadien des forêts au (819) 997-1107. Si vous désirez obtenir des copies additionnelles, veuillez écrire à l'adresse suivante: Service canadien des forêts, Direction des communications, Ottawa K1A 1 G5

\section{Notes explicatives}

- Tout en reconnaissant l'importance des divers programmes offerts au niveau du secondaire, seuls les programmes post-secondaires d'une durée d'au moins un an sont inclus pour l'usage de ce document. Les programmes sont présentés par province et sous forme de tableau indiquant les types de programmes et l'endroit où ils sont offerts. Les différentes options d'un programme ne sont normalement pas incluses.

- Les nombres qui apparaissent au-dessus des tableaux correspondent aux universités et écoles offrant des programmes relatifs au secteur forestier. 


\begin{tabular}{lll}
\hline \multicolumn{3}{c}{$\begin{array}{c}\text { Forest Sector-related Programs/ } \\
\text { Programmes relatifs au secteur forestier } \\
\text { Newfoundland/Terre-neuve }\end{array}$} \\
\hline \multicolumn{1}{c}{1} & 2 \\
\hline $\begin{array}{l}\text { Forestry Technology } \\
\text { Pre-forestry }\end{array}$ & $\mathrm{d}$ & $\mathrm{P}$ \\
\hline
\end{tabular}

Codes

d - Diploma (three years

P-Pre-program

Upon successful completion of the program in Pre-forestry, students will be admitted with advanced standing to the five year Forestry Degree program of the University of New Brunswick.

\begin{tabular}{|c|c|}
\hline \multicolumn{2}{|c|}{$\begin{array}{l}\text { Universities and Schools Offering Forest } \\
\text { Sector-related Programs/ } \\
\text { Universités et écoles offrant des programmes } \\
\text { relatifs au secteur forestier }\end{array}$} \\
\hline \multicolumn{2}{|c|}{ Newfoundland/Terre-neuve } \\
\hline $\begin{array}{l}\text { 1) Fisher Technical Institute } \\
\text { P.O. Box } 822 \\
\text { Corner Brook, Nfld. } \\
\text { A2H } 6 \mathrm{H} 6 \\
\text { (709) } 637-2501\end{array}$ & $\begin{array}{l}\text { 2) Memorial University of } \\
\text { Newfoundland } \\
\text { Department of Forestry } \\
\text { Mr. Cook } \\
\text { St. John's, Nfld. } \\
\text { A1C } 5 \text { S7 } \\
\text { (709) } 737-8812\end{array}$ \\
\hline
\end{tabular}

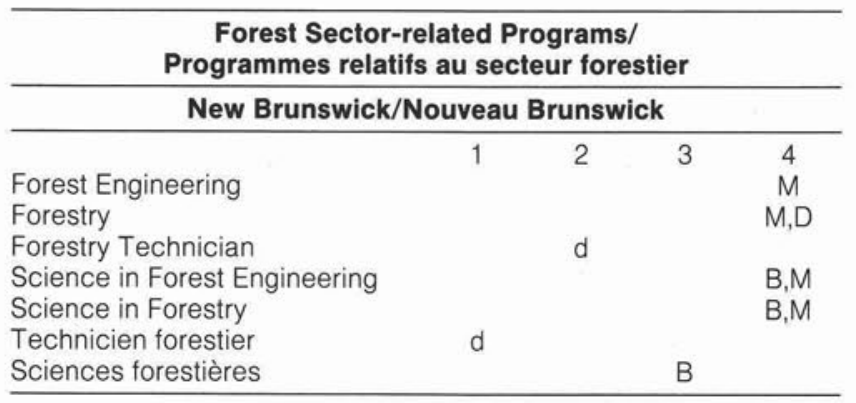

Codes

B - Bachelor's degree (five years)/baccaalauréat (cinq ans)

d-Diploma (one year)/diplôme (un an)

D - Doctorate degree (minimum of three-years)/doctorat (minimum de trois ans)

M - Master's degree (two years)/maitrise (deux ans)

\begin{tabular}{lc}
\hline \multicolumn{1}{c}{$\begin{array}{c}\text { Universities and Schools Offering Forest Sector-related Programs/ } \\
\text { Universités et écoles offrant des programmes relatifs au secteur forestier } \\
\text { New Brunswick/Nouveau-Brunswick }\end{array}$} \\
\hline 1) Ecole des gardes forestiers & 3) Université de Moncton \\
725 rue du Collège & Ecole de sciences forestières \\
C.P. 1 & Centre universitaire Saint-Louis Maillet \\
Bathurst, NB & 165, boulevard Hébert \\
E2A 3Z2 & Edmundston, NB \\
(506) 546-4176 & E3V 2S8 \\
2) Maritime Forest Ranger School & (506) 735-8804, poste 340 \\
Regent Street Extension & 4) University of New Brunswick \\
R.R. No. 10 & Department of Forestry Resources \\
Fredericton, NB & Faculty of Forestry \\
E3B 6H6 & Bag Service 44555 \\
(506) 458-0199 & Fredericton, NB \\
& E3B 6C2 \\
& (506) 453-4501 \\
\hline
\end{tabular}




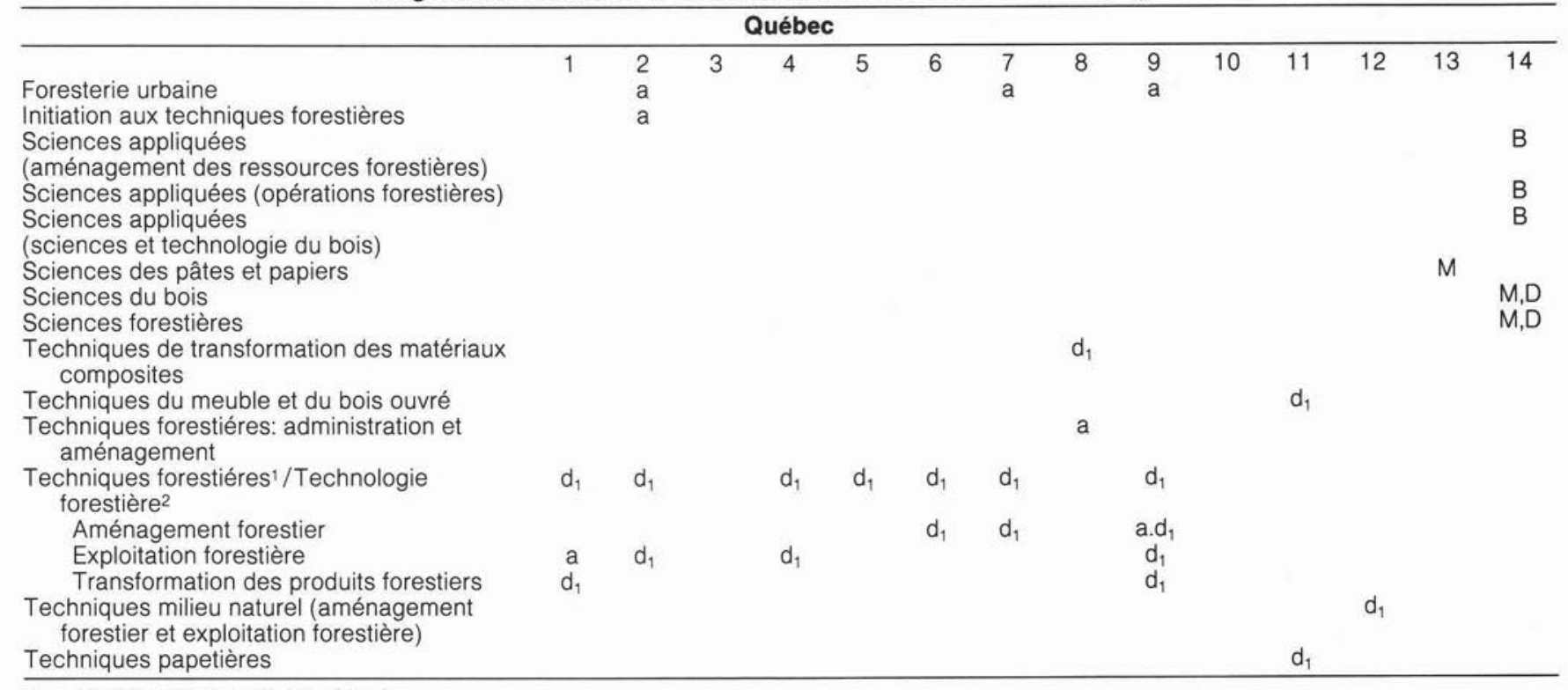

a - Attestation d'études collégiales (un an)

$\mathrm{B}$ - Baccalauréat (trois à quatre ans)

c - Certificat d'études collégiales (deux ans)

$d_{1}$ - Diplôme d'étude colégiales (trois ans)

D - Doctorat (trois ans)

M - Maitrise (un an et demi à deux ans)

1) Tronc commun de deux ans pour les étudiants gradués du secondaire V. A la troisième année, choix d'une spécialisation en Aménagement forestier ou Exploitation forestière ou Transformation des produits forestiers.

2) Tronc commun de deux ans pour les étudiants gradués du secondaire $V$ et qui ont déjà une formation dans le secteur forestier. A la troisième année, choix d'une spécialisation en Aménagement forestier ou Exploitation forestière ou Transformation des produits forestiers.

\begin{tabular}{c}
\hline $\begin{array}{c}\text { Universités et écoles offrant des programmes relatifs au secteur forestier/ } \\
\text { Universities and Schools Offering Forest Sector-related Programs }\end{array}$ \\
\hline Québec
\end{tabular}

1) Collège de l'Abitibi-

Témiscamingue

425 , boulevard du Collège

C.P. 1500

Rouyn, Québec

J9X 5E5

(819) 762-0931

2) Collège de Chicoutimi

534, rue Jacques-Cartier est

C.P. 1148

Chicoutimi, Québec

G7H 1 Z6

(418) $549-9520$

3) Collège de Drummondville

960, rue Saint-Georges

Drummondville, Québec

J2C 6 A2

(819) 478-4671

4) Collège de la Gaspésie et des îles

96, rue Jacques-Cartier

C.P. 590

Gaspé, Québec

GOC 1R0

(418) $385-2241$

5) Collège de Baie Comeau 537, boulevard Blanche Baie Comeau, Québec

G5C 2B2

(418) 589-5707
6) Collège John Abbot

C.P. 2000

Saint-Anne-de-Bellevue,

Québec

H9X 3L9

(514) 457-6610

7) Collège de Rimouski

60 , rue de l'Eveché ouest

Rimouski, Québec

G5L $4 \mathrm{H} 6$

(418) $723-1880$

8) Collège de Saint-Jérôme

455 , rue Fournier

Saint-Jérôme (Terrebonne),

Québec

J7Z 4V2

(514) 436-1580

9) Collège de Sainte-Foy

2410, chemin Sainte-Foy

Sainte-Foy, Québec

G1V 1T3

(418) $659-6600$
10) Collège de Trois-Rivières

Pavillon de la Papeterie 3500 , rue De Coural C.P. 97

Trois-Rivières, Québec G9A 5E6

(819) 376-1721

11) Collège de Victoriaville 475 , rue Notre-Dame est Victoriaville, Québec G6P 4B3

(819) 758-6401

12) Collège de St-Félicien 1105, boulevard Hamel St-Félicien, Québec G8K 2R8

(418) 679-5412

13) Université du Québec à Trois-Rivières Bureau du registraire C.P. 500

Trois-Rivières, Québec

G9A $5 \mathrm{H} 7$

(819) 376-5045

14) Université Laval

Faculté de foresterie et géodésie

C.P. 2208

Québec, Québec

G1K 7P4

(418) 656-3053 


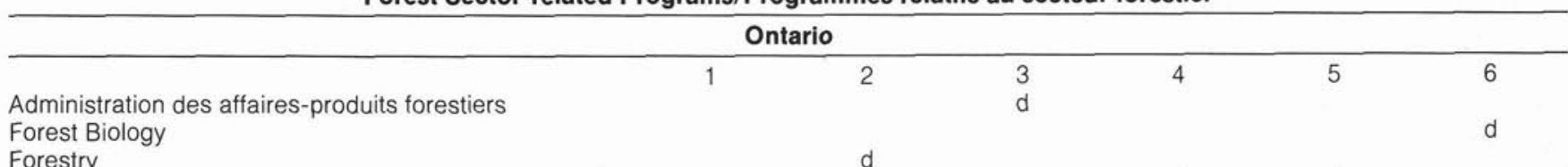

\section{Forest Biology}

Forestry

Forestry (Fish and Wildlife) Technology2

Forestry (Parks \& Recreation) Technology ${ }^{2}$

Forest Recreation Technician

Forestry Technician'

Forestry Technology2

Natural Resources Technology - Law Enforcement

Resource Management

Science (Pulp \& Paper) Engineering Technology

Science in Forestry

Techniques forestières

Wood Products Management

Wood Science

B - Bachelor's degree (four years)/baccalauréat (quatre ans)

d- Diploma (programs are usually of four semesters' duration or more)/diplôme (les programmes durent normalement quatre semestres ou plus)

D - Doctorate degree (three and a half years)/doctorat (trois ans et demie)

M - Master's degree (two and a half years)/maitrise (deux ans et demie)

1. A typical full-time technician program lasts four semesters or two years. Une programme à temps plein de formation de technicien dure généralement quatre semestres ou deux ans.

2. A typical full-time technologist training program takes six semesters or three years. The Forestry Technician program is a pre-requisite and counts for two years./Un programme à temps plein de formation de technicien specialiste dure généralement six semestres ou trois ans. Le programme Technicien forestier est un pré-requis et compte pour deux ans. d

B,M,D d

Universities and Schools Offering Forest Sector-related Programs/ Universités et écoles offrant des programmes relatifs au secteur forestier

\section{Ontario}

1) Algonquin College of Applied Arts and Technology/ Collège Algonquin d'arts appliqués et de technologie

School of Renfrew County Campus/

Ecole du compté de Renfrew

315, Pembroke Street East/

rue Pembroke est

Pembroke, Ontario

K8A 3K2

(613) 735-4708

2) Lakehead University

The School of Forestry

Thunder Bay, Ontario

K7B 5E1

(807) $343-8110$

3) Northern College/Collège

Northern d'arts appliqués et de technologie

Campus Kirkland Lake Campus

Registrar and Director of Student Services/Registraire

140 Government Rd. E./140 est, chemin Gouvernement

Kirkland Lake, Ontario

P2N 3L8

(705) 567-9291
4) Sault College of Applied Arts and Technology Registrar's Office

P.O. Box 60

Sault Ste. Marie, Ontario

P6A 5L3

(705) 949-2050

5) Sir Sandford Fleming College of Applied Arts and Technology Admissions Office

School of Natural Resources

Frost Campus

P.O. Box 8000

Lindsay, Ontario

K9V 5E6

(705) 324-9144

6) University of Toronto

Faculty of Forestry

203 College Street

Toronto, Ontario

M5S 1 A1

(416) $978-6152$ 
Forest Sector-related Programs/

Programmes relatifs au secteur forestier

\section{Manitoba}

Natural Resources Management

Codes

d - Diploma (two years)
Universities and Schools Offering Forest Sector-related Programs/

Universités et écoles offrant des programmes relatifs au secteur forestier

Manitoba

1) Keewatin Community College

P.O. Box 3000

The Pas, Manitoba

R9A 1 M7

(204) 623-3416

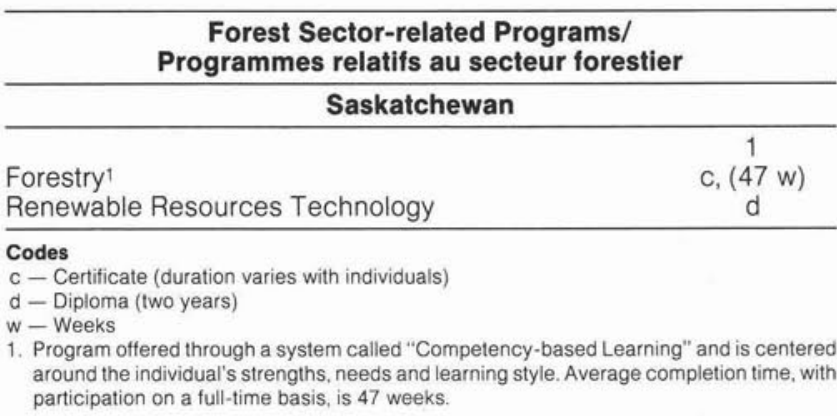

Forest Sector-related Programs/ Saskatchewan

\section{Forest Science/Technology}

Forest Technology

Forestry

Renewable Resource Management

Forest Sector-related Programs/Programmes relatifs au secteur forestier

\author{
B - Bachelor's degree (four years) \\ d - Diploma (two years) \\ D - Doctorate degree (two to three years) \\ M-Master's degree (two to three years) \\ y - University transfer: First year equivalent towards a bachelor's degree at the University of Alberta
}

$\begin{array}{r}\text { Universities and Schools Offering Forest } \\ \text { Sector-related Programs/ }\end{array}$
Universités et écoles offrant des programmes
relatifs au secteur forestier

y University transter: First year equivient towards a bachelor's degreeat the University of Alberta

Universities and Schools Offering Forest Sector-related Programs/ Universités et écoles offrant des programmes relatifs au secteur forestier

Lethbridge, Alberta

\section{Alberta}
1) Grande Prairie Regional College
The Registrar
10726-106 Avenue
Grande Prairie, Alberta
T8V 4 C4
(403) 539-2919
5) Red Deer College
The Registrar
Box 5005
Red Deer, Alberta
T4N $5 \mathrm{H} 5$
(403) $342-3300$

2) Lethbridge Community College

The Registrar

3000 College Drive South

T1K $1 \mathrm{~L} 6$

(403) $320-3200$

3) Medicine Hat College

The Registrar

299 College Drive

Medicine Hat, Alberta

T1A 3 Y 6

(403) 529-3811

4) Mount Royal College

The Registrar

4825 Richard Road SW

Calgary, Alberta

T3E 6 K 6

(403) 240-6363
6) Camrose Lutheran College

Office of Admissions

Camrose, Alberta

T4V 2R3

(403) 679-1100

7) Concordia College

The Registrar

7128 Ada Boulevard

Edmonton, Alberta

T5B 4E4

(403) $479-8481$

8) The King's College

The Director of Student Affairs

10766-97 Street

Edmonton, Alberta

$\mathrm{T} 5 \mathrm{H} 2 \mathrm{M} 1$

(403) 428-0727
9) Northern Alberta Institute of Technology (NAIT)

The Registrar

11762-106 Street

Edmonton, Alberta

T5G 2R1

(403) $471-7411$

10) The University of Alberta Agriculture Forestry Centre

Faculty of Agriculture and Forestry

Edmonton, Alberta

T6G 2P5

(403) 432-4931

11) The University of Calgary 2500 University Drive NW

Calgary, Alberta

T2N 1 N4

(403) 284-6645

12) The University of Lethbridge

The Registrar

4401 University Drive

Lethbridge, Alberta

T1K $3 \mathrm{M} 4$

(403) 329-2231 
Applied Sciences (Forest Engineering)

Chemical Sciences (Pulp and Paper)

Forest Products

Forest Resources Technology

Forestry

Greenhouse Technology

Lumber and Plywood Technology

Science of Forestry

Science (Forestry)

Wildland Recreation Technology

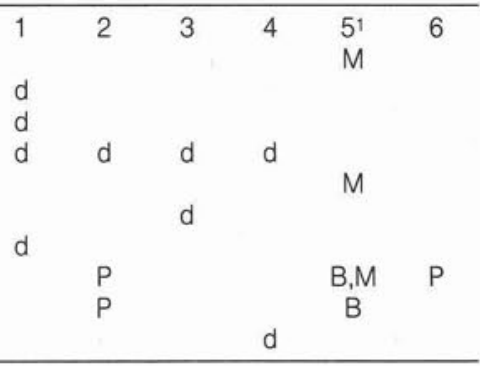

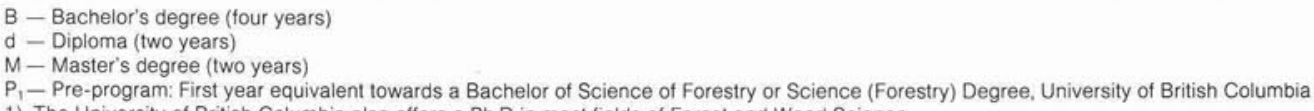

1) The University of British Columbia also offers a Ph.D in most fields of Forest and Wood Science.

Universities and Schools Offering Forest Sector-related Programs/ Universités et écoles offrant des programmes relatifs au secteur forestier

British Columbia/Colombie-britannique

1) British Columbia Institute of Technology (BCIT)

Department of Forest Resources

2700 Willingdon Avenue

Burnaby, B.C.

V5 $53 \mathrm{H} 2$

(604) 434-1610

2) College of New Caledonia

3330-22 Avenue

Prince George, B.C

V2N $1 \mathrm{P} 8$

(604) 562-2131

3) Malaspina College

900 Fifth Street

Nanaimo, B.C.

V9R 5S5

(604) 753-3245
4) Selkirk College

P.O. Box 1200

Castlegar, B.C

V1N 3J1

(604) 365-7292

5) The University of British Columbia (UBCO

Faculty of Forestry

204-2075 Westbrook Mall

Vancouver, B.C.

V6T 1 Z2

(604) 228-2727

6) University of Victoria (UVic)

Admissions Services

Box 1700

Victoria, B.C.

V8W $2 Y 2$

(604) $721-7211$
Forest Sector-related Programs/

Programmes relatifs au secteur forestier

Northwest Territories/Territoires du nord-ouest

Renewable Resources Technology

d

\section{Codes}

d - Diploma (two years)
Forest Sector-related Programs/

Programmes relatifs au secteur forestier

Northwest Territories/Territoires du nord-ouest

1) Arctic College

Thebacha Campus

Registrar

P.O. Box 600

Fort Smith, Northwest Territories

XOA OP6

(403) $872-7509$ 